\title{
Comparison of initial outcomes between minimally invasive right minithoracotomy and standard median sternotomy in patients undergoing isolated surgical closures of atrial septal defects.
}

Sumsul Arif Mohammad Musa ${ }^{1}$, Mauin Uddin ${ }^{*}$, Syed Al Nahian ${ }^{3}$ Mohammad Parvez Ahmed Saikat Das Gupta ${ }^{5}$, Rubana Forhad ${ }^{6}$, Prasanta Kumar Chanda ${ }^{7}$, Farooque Ahmed ${ }^{8}$, Mohammad Mostafizur Rahman Miah'

${ }^{1}$ National Heart Foundation Hospital and Research Institute, Dhaka, Bangladesh

${ }^{2}$ New Cross Hospital, Wolverhampton, United Kingdom

${ }^{3}$ Department of Cardiothoracic-Surgery, Liverpool Heart and Chest Hospital, Liverpool, United Kingdom

${ }^{4}$ University Hospital Coventry and Warwickshire, Coventry, United Kingdom

${ }^{5}$ Department of Cardiac Surgery, National Heart Foundation Hospital and Research Institute, Dhaka, Bangladesh

${ }^{6}$ Department of Reproductive and Child Health, Bangladesh University of Health Sciences, Dhaka, Bangladesh

${ }^{7}$ Department of Cardiac Surgery, National Heart Foundation Hospital and Research Institute, Dhaka, Bangladesh

${ }^{8}$ National Hear Foundation Hospital and Research Institute, Dhaka, Bangladesh

${ }^{9}$ National Institute of Cardiovascular Diseases, Dhaka, Bangladesh

\begin{abstract}
Background: An atrial septal defect (ASD) can be closed via percutaneous approach and with surgical closure using either the standard median sternotomy or a minimally invasive procedure such as minithoracotmy. In this study, we evaluated the outcomes of using the median sternotomy vs. the right minithoracotomy in isolated closures of ASDs.

Methods: This prospective observational study included 44 patients who underwent isolated ASD closures in the department of cardiac surgery at the National Heart Foundation Hospital and Research Institute (NHFH and RI) in Dhaka, Bangladesh, from July 2014 to June 2016. After fulfilling the enrolment criteria, participants were divided into two equal groups of 22 patients. ASD closure was performed on Group 1 using the minimally invasive right minithoracotomy, while the same procedure was performed on Group 2 using the standard median sternotomy.

Results: The mean total operative time (in minutes) required was $179.45 \pm 26.79$ for Group 1 and $154.72 \pm 26.15$ for Group 2. The mean aortic cross-clamp (ACC) time (in minutes) and cardiopulmonary bypass (CPB) time (in minutes) were significantly increased in Group 1 compared to Group $2(p=<0.05)$. Furthermore, the mean \pm SD of the length of incision (in centimetres) and duration of endotracheal intubation (in hours) were significantly higher in Group 2 than Group 1 (p<0.001). While the duration of postoperative hospital stays (in days) and pain score (visual analogue scale score) was significantly higher in Group 2 than Group 1. No residual shunt was found in patients of either group.

Conclusion: Our findings shows some advantages in group 1 (right minithoracotomy) population including the reduced length of incision, the shorter duration of endotracheal intubation, less postoperative blood loss, fewer transfusion requirements, minimal postoperative pain and the shorter duration of hospital stay. In addition, patients in Group 1 reported smaller scars, as expected, which contributes to better overall patient satisfaction.
\end{abstract}

Keywords: Minimally invasive surgical procedures, Sternotomy, Congenital heart defects.

Accepted on May 11, 2020

\section{Introduction}

Atrial septal defects (ASDs) are one of the most common congenital anomalies. While a small variety of ASDs may close naturally, many necessitate intervention. Percutaneous closure using the Amplatzer device is possible for secundum ASDs, which retain a sufficient rim of tissue around the defect. Conversely, the surgical closure of ASD is traditionally accomplished through a median sternotomy, cardiopulmonary bypass (CPB) and cardioplegic arrest of the heart; however, the unpleasant cosmetic outcome and possible complications of median sternotomy are occasionally distressing to patients [1]. It would therefore be prudent for surgeons to compare the initial postoperative outcomes of patients who have undergone ASD closure via right minithoracotomy, under direct vision, 
Citation: Musa SAM, Uddin M, Nahian SA, et al. Comparison of initial outcomes between minimally invasive right minithoracotomy and standard median sternotomy in patients undergoing isolated surgical closures of atrial septal defects. Curr Trend Cardiol 2020; $4(1): 18-23$

with the same outcomes of those who have undergone ASD closure via the standard median sternotomy to better understand the safety and effectiveness of both surgical procedures [2].

\section{Rationale}

Atrial septal defect closure is a surgical procedure often performed at the National Heart Foundation Hospital and Research Institute (NHFH and RI) in Dhaka, Bangladesh. For almost all ASD closure cases, the median sternotomy has been the favoured method; in fact, using the right minithoracotomy to close ASDs is not a routine practice here. It is rather an innovative way to reform cardiac surgery in this country considering cardiac surgeons' increasing interest in performing minimally invasive procedures, in addition to the rising patient demand for less-invasive procedures.

\section{Hypothesis}

Atrial septal defect closure using the right minithoracotomy, under direct vision, is predicted to have less postoperative morbidity compared to the standard median sternotomy.

\section{Methods}

This comparative study was conducted at the NHFH and RI in Dhaka, Bangladesh, between July 2014 and June 2016. It involved all patients who underwent isolated ASD closures using either the right minithoracotomy or the standard median sternotomy.

\section{Ethical issues}

The ethical review committee of the NHFH and RI approved this project. All participants were verbally informed of the study's design and purpose, as well as their right to withdraw from the study at any time for any reason. Written consent was obtained from each participant.

\section{Sampling technique}

Purposive and convenience sampling were used for all consecutive patients who fulfilled the enrolment criteria.

\section{Selection criteria}

Patients who were undergoing isolated surgical ASD closure for the first time were included in the study. Patients exhibiting the following characteristics were excluded from the study: decreased left ventricular ejection fraction $(<30 \%)$ and those associated with other congenital heart disease.

For the purposes of this study, 44 patients undergoing isolated surgical ASD closure were divided into two equal groups of 22 participants to be studied. The sample size of both groups was determined using the following formula:

$\mathrm{n}=2 \sigma^{2} / \Delta^{2}(\mathrm{z} \alpha+\mathrm{z} \beta)^{2}$

Since $n=22$, the total sample size was $44(22 \times 2=44)$.

\section{Operative technique}

At the NHFH and RI, the current preferred method for minimally invasive ASD closure involves the fourth intercostal space minithoracotomy incision, which provides improved cosmesis with a direct view from a lateral perspective into the right atrium and interatrial septum. For each participant who underwent this procedure, a skin incision of $6-8 \mathrm{~cm}$ was made over the chosen interspace, and the intercostal incision was extended beyond the skin incision, which allows a retractor to spread the ribs while minimizing the risk of breaking them. A 3 $\mathrm{cm}$ incision was also made just underneath the right inguinal fold; the anterior femoral artery and vein were thus exposed with minimal dissection. After a full dose of heparin a femoral arterial cannula was advanced into the proximal iliac artery using the Seldinger technique: a femoral venous cannula was passed over a guidewire to the right atrium under TEE visualization. Once cannulation had been completed, the patient was placed on $\mathrm{CPB}$. When the pericardium was opened anterior to the phrenic nerve, multiple pericardial retention sutures were placed anteriorly and posteriorly to retract and define the access to Sondergaard's groove and obtain access to the aorta. A CP cannula was then inserted into the ascending aorta. Furthermore, a Cosgrove flexible aortic cross-clamp (Figure 1) was applied, after which cardioplegic arrest of heart was achieved and Sondergaard's groove was opened.

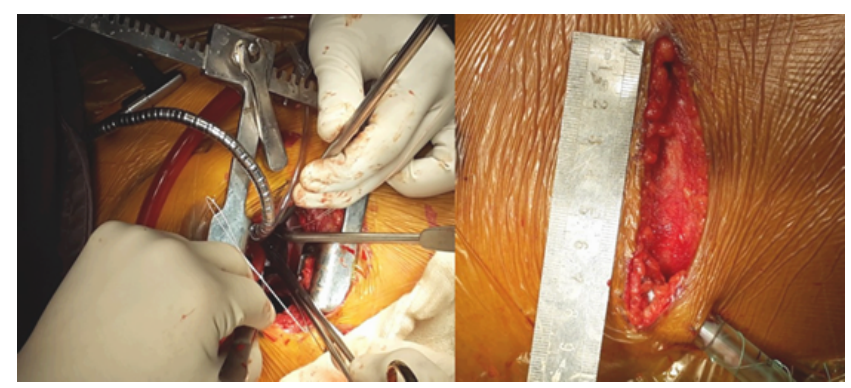

Figure 1. (A)External rib retractor with flexible aortic cross clamp (B) Incision site.

A floppy vent cardiotomy sucker was dropped into the open left pulmonary veins, and a retractor was used to elevate the right atrial wall and expose the ASD. Direct visualization was found to be excellent with this procedure. Atrial septal defect closure was performed using a glutaraldehyde-treated pericardial patchviaconventional instruments, as well as the continuous suture technique. The right atrial closure was then completed. The cross-clamp was released after proper deairing, and when the patient had been weaned from the CPB, decannulation was conducted as well. Finally, the chest closed in layers keeping chest drain in place.

\section{Results}

\section{Preoperative factors}

The mean age (in years) of the study population was $29.68 \pm$ 12.37 for Group 1 and $28.57 \pm 11.29$ for Group 2. In Group 1, $10(45.4 \%)$ patients were male, and $12(55.6 \%)$ patients were female; in Group 2, $11(50.0 \%)$ patients were male, and 11 
$(50.0 \%)$ patients were female. No statistically significant $(p>0.05)$ differences in patient characteristics such as ejection fraction, LA diameter, pulmonary artery systolic pressure (PASP) and atrial fibrillation was found between these two groups (Table 1).

Table 1. Distribution of patients in both groups based on preoperative patient characteristics.

\begin{tabular}{|c|c|c|c|}
\hline $\begin{array}{l}\text { Preoperative patient } \\
\text { characteristics }\end{array}$ & Group & & $\begin{array}{c}p \\
\text { value }\end{array}$ \\
\hline & Group-1 & Group-2 & \\
\hline & $\begin{array}{l}\text { (Right mini } \\
\text { thoracotomy) }\end{array}$ & $\begin{array}{c}\text { (Median } \\
\text { sternotomy) }\end{array}$ & \\
\hline & Mean \pm SD & Mean \pm SD & \\
\hline Age (Mean \pm SD) & $29.68 \pm 12.37$ & $28.57 \pm 11.29$ & 0.75 \\
\hline \multicolumn{4}{|l|}{ Sex } \\
\hline - $\quad$ Male & $10(45.4)$ & $11(50.0 \%)$ & 1 \\
\hline - $\quad$ Female & $12(55.6)$ & $11(50.0 \%)$ & \\
\hline Ejection fraction & $58.47 \pm 5.26$ & $56.72 \pm 7.39$ & 0.37 \\
\hline LA diameter & $53.55 \pm 3.27$ & $53.86 \pm 5.01$ & 0.92 \\
\hline
\end{tabular}

\begin{tabular}{|l|l|l|l|}
\hline $\begin{array}{l}\text { Pulmonary artery systolic } \\
\text { pressure (PASP) }\end{array}$ & $39.72 \pm 10.73$ & $40.0 \pm 14.27$ & 0.94 \\
\hline Atrial fibrillation & $4(18.2 \%)$ & $3(13.6 \%)$ & $1^{\text {** }}$ \\
\hline
\end{tabular}

*An independent sample t-test was conducted to measure the level of significance.

**Fisher's exact test was conducted to measure the level of significance.

\#A figure within parentheses indicates in percentage.

\section{Perioperative factors}

The mean total operative time (in minutes) was $179.45 \pm 26.79$ for Group 1 and $154.72 \pm 26.15$ for Group 2. The difference between these two groups was therefore significant $(p=0.004)$. Moreover, the mean aortic cross-clamp (ACC) time (in minutes) was significantly increased in Group 1 compared to Group 2 (43.28 \pm 21.75 vs. $21.17 \pm 11.25)(\mathrm{p}=0.018)$. The mean CPB time (in minutes) was $76.26 \pm 26.51$ for Group 1 and $46.84 \pm 25.72$ for Group 2. The mean length of incision (in centimeters) was $7.63 \pm 0.69$ for Group 1 and $22.62 \pm 1.93$ for Group 2, which was found to be statistically significant (Tables 2 and 3 ).

Table 2. Distribution of patients of both groups by operative parameters.

\begin{tabular}{|c|c|c|c|}
\hline \multirow[t]{4}{*}{ Operative Variables } & \multicolumn{2}{|c|}{ Group } & \multirow[t]{4}{*}{$\mathrm{p}$ value } \\
\hline & Group-1 & Group-2 & \\
\hline & (Right mini thoracotomy) & (Median sternotomy) & \\
\hline & Mean \pm SD & Mean \pm SD & \\
\hline Total operative time (in minutes) & $179.45 \pm 26.79$ & $154.72 \pm 26.15$ & 0.003 \\
\hline Aortic Cross Clamp (ACC) time (in minutes) & $43.28 \pm 21.75$ & $21.17 \pm 11.25$ & 0.001 \\
\hline Cardio-pulmonary bypass (CPB) time (in minutes) & $76.26 \pm 26.51$ & $46.84 \pm 25.72$ & 0.006 \\
\hline Length of incision $(\mathrm{cm})$ & $7.63 \pm 0.69$ & $22.62 \pm 1.93$ & $<0.001^{*}$ \\
\hline
\end{tabular}

*A t-test was conducted to measure the level of significance.

Table 3. Distribution of patients in both groups based on postoperative outcomes.

\begin{tabular}{|c|c|c|c|}
\hline \multirow[t]{4}{*}{ Post-operative Outcome variables } & \multicolumn{2}{|c|}{ Group } & \multirow[t]{4}{*}{ p value } \\
\hline & Group-1 & Group-2 & \\
\hline & (Right mini thoracotomy) & (Median sternotomy) & \\
\hline & Mean \pm SD & Mean \pm SD & \\
\hline Duration of endotracheal incubation (hours) & $11.64 \pm 2.01$ & $15.05 \pm 4.91$ & 0.003 \\
\hline Duration of ICU stay (hours) & $39.36 \pm 12.95$ & $44.55 \pm 12.97$ & 0.191 \\
\hline Post-operative hospital stay (days) & $6.91 \pm 1.44$ & $8.00 \pm 1.90$ & 0.03 \\
\hline Pain (visual analogue scale score 1-10) & $3.19 \pm 0.52$ & $5.49 \pm 0.64$ & $<0.001^{*}$ \\
\hline ** No. of patient transfused & $4(22.7 \%)$ & $9(45.5 \%)$ & 0.18 \\
\hline Reoperation for bleeding & $0(0.0)$ & $0(0.0)$ & -- \\
\hline
\end{tabular}


Citation: Musa SAM, Uddin M, Nahian SA, et al. Comparison of initial outcomes between minimally invasive right minithoracotomy and standard median sternotomy in patients undergoing isolated surgical closures of atrial septal defects. Curr Trend Cardiol 2020; 4(1):18-23.

\begin{tabular}{|l|l|l|l|}
\hline *Amount of blood transfused (Mean \pm SD) & $375 \pm 100.5$ & $470 \pm 106.12$ & 0.004 \\
\hline *Postoperative blood loss(ml) (Mean \pm SD) & $198.24 \pm 77.25$ & $351.45 \pm 120.92$ & $<0.001$ \\
\hline Stroke & $0(0.0)$ & $0(0.0)$ & -- \\
\hline Renal Failure & $0(0.0)$ & $0(0.0)$ & -- \\
\hline Atrial Fibrillation & $1(4.5)$ & $2(9.1)$ & $1.000^{*}$ \\
\hline
\end{tabular}

\section{Follow-up}

During the follow-up, atrial fibrillation was found in one $(4.5 \%)$ case in Group 1 and two cases $(9.1 \%)$ in Group 2. No case of stroke or renal failure was reported for either group. Moreover, no deep wound infection emerged in either group; however, superficial wound infections were found in one (4.5\%) patient in Group 1 and two (9.1\%) patients in Group 2. This study revealed the following echocardiography findings for the study population after one month of follow-up: the mean ejection fraction was $55.74 \pm 4.03$ for the right minithoracotomy and $54.27 \pm 6.12$ for the median sternotomy; the mean LA diameter was $46.32 \pm 2.77$ for the right minithoracotomy and $46.36 \pm 2.77$ for the median sternotomy; and the mean PASP was $36.24 \pm 7.37$ for the right minithoracotomy and $39.74 \pm 8.91$ for the median sternotomy. No residual shunt was found in patients of either group.

\section{Discussion}

Minimally invasive approach of ASD closure can be achieved by several techniques for instance partial sternotomy, right parasternal mini-incision, right anterolateral thoracotomy, right posterolateral thoracotomy, video-assisted mini-thoracotomy, robot-assisted surgery, and total thoracoscopic surgery without robotic assistance [3-7]. MICS reveals a cosmetic advantage. However, MICS has the negative aspects of a confined operative area and technical difficulties in peripheral cannulation and aortic cross-clamping. Several studies of MICS for ASD closure have been performed, and most effects have published similar outcomes between the 2 groups [8-11]. Right anterolateral thoracotomy has the benefit of offering a direct field of vision to the right atrium, which may additionally be incised and approached for ASD. Therefore, the skin incision is minimized and adjusted to the measurement required for ASD closure.

Our results revealed that the MICS group experienced significantly longer operation times, CPB times, and ACC times. Contributing factors are variations in femoral vessel development, peripheral cannulation and restricted operative field. On the contrary post-operative recovery was faster because of less ICU stay, minimal pain and early mobility. There was no conversion to full sternotomy in the MICS group, which means there was no failure of the minimally invasive procedure. There were also no additional complications because of femoral cannulation such as seroma, lymphocele, wound infection, or pseudoaneurysm in the MICS group. No residual shunt was identified in either of the group.

\section{Limitations}

This study was limited due to the following factors: it was a single-centre study, it used a purposive non-random sampling method, its sample size was small, and no data was collected after the one-month follow-up. In fact, no information regarding the participants' postoperative quality of life, including their satisfaction with the procedure, return to work, level of discomfort and performance of daily activities following their ASD closures through right minithoracotomy or median sternotomy, was even mentioned.

\section{Recommendations}

Based on the findings of this study, future research should involve

- A large-scale multicentre study.

- A long-term follow-up.

- $\quad$ Sufficient training for and expertise in using the minimally invasive right thoracotomy in cardiac surgery; and

- A modern surgical instrument for following the meticulous surgical procedure.

\section{Conclusion}

This study confirms that ASD closure can be performed using a right minithoracotomy without compromising the patient's safety. In addition, its findings, and Group 1 in particular, suggest some advantages to using the right minithoracotomy, including the reduced length of incision, the shorter duration of endotracheal intubation, less postoperative blood loss, fewer transfusion requirements, minimal postoperative pain, and the shorter duration of hospital stay. Patients in Group 1 also reported smaller scars, as expected, which contributes to better overall patient satisfaction.

\section{Key Clinical Message}

ASD closure by minithoracotomy can be a safe and affordable option even for a developing country. Although it requires expertise.

\section{Author Contribution}

\section{Conception and design of the research}

Dr. Sumsul Arif Mohammad Musa

Dr. Mauin Uddin 
Dr. Prasanta Kumar Chanda

\section{Collection of the data}

Dr. Mauin Uddin

Dr. SumsulArif Mohammad Musa

Dr. Mohammad Parvez Ahmed

Analysis and interpretation of the data

Dr. Mauin Uddin

Dr. Saikat Das Gupta

Dr. Rubana Forhad

Dr. Mohammad Mostafizur Rahman

Dr. Mohammad Parvez Ahmed

\section{Analysis and interpretation of the data}

Dr. Mauin Uddin

Dr. Saikat Das Gupta

Dr. Rubana Forhad

Dr. Mohammad Mostafizur Rahman

Dr. Mohammad Parvez Ahmed

\section{Statistical analysis}

Dr. RubanaForhad

Dr. Saikat Das Gupta

Dr. Sumsul Arif Mohammad Musa

Dr. Mohammad Mostafizur Rahman

\section{Drafting the manuscript}

Dr. Mauin Uddin

Dr. Sumsul Arif Mohammad Musa

Dr. Mohammad Mostafizur Rahman

Dr. Syed Al Nahian

\section{Critical revision of the manuscript}

Dr. Mauin Uddin

Dr. Mohammad Mostafizur Rahman

Dr. Saikat Das Gupta

Dr. Syed Al Nahian

\section{Final approval of the manuscript}

Dr. Prasanta Kumar Chanda

\section{Obtained funding}

Dr. Prasanta Kumar Chanda
Dr. Farooque Ahmed

\section{Overall responsibility}

Dr. Sumsul Arif Mohammad Musa

Dr. Prasanta Kumar Chanda

Dr. Farooque Ahmed

\section{References}

1. Black MD, Freedom RM. Minimally invasive repair of atrial septal defects. Ann Thorac Surg. 1998;65(3):765-67.

2. Cremer JT, Boning A, Anssar MB, et al. Different approaches for minimally invasive closure of atrial septal defects. Ann Thorac Surg. 1999;67(6):1648-52.

3.Grinda JM, Folliguet TA, Dervanian P, et al. Right anterolateral thoracotomy for repair of atrial septal defect. Ann Thorac Surg. 1996;62(1):175-178.

4. Kim JE, Jung SH, Kim GS, et al. Surgical outcomes of congenital atrial septal defect using da VinciTM Surgical Robot System. Korean J Thorac Cardiovasc Surg. 2013;46 (2):93-7.

5.Lancaster, LL,Mavroudis, C, Rees AH, et al. Surgical approach to atrial septal defect in the female. Right thoracotomy vs. sternotomy. Am Surg. 1990; 56(4):218-21.

6. Massetti M,BabatasiG, Rossi A, et al. Operation for atrial septal defect through a right anterolateral thoracotomy: current outcome. Ann Thorac Surg.1996;62(4):1100-03.

7. Ma ZS, Dong MF, Yin QY, et al. Totally thoracoscopic repair of atrial septal defect without robotic assistance: a single-center experience. J Thorac Cardiovasc Surg. 2011;141(6):1380-83.

8. OwaisTA, Farber G, Garbade J, et al. Excision of a left atrial myxoma via a minimally-invasive technique: a possible routine access. Interactive CardioVascular and Thoracic Surgery. 2011; 12(5):875-77.

9.Poyrazoglu HH, Avsar MK, Demir S, et al. Atrial septal defect closure: comparison of vertical axillary minithoracotomy and median sternotomy. Korean J ThoracCardiovascSurg. 2013;46(5):340-45.

10.Ryan WH, Cheirif J, Dewey TM, et al. Safety and efficacy of minimally invasive atrial septal defect closure. Ann ThoracSurg. 2003;75(5):1532-34.

11. Sabate Rotes A, Burkhart HM, Suri RM, et al. Minimally invasive video-assisted surgical closure of atrial septal defects: a safe approach. World J PediatrCongenit Heart Surg. 2014;5(4):527-33.

\section{*Correspondence to}

Mauin Uddin

New Cross Hospital

Wolverhampton

United Kingdom

E-mail:mu_ahmed07@yahoo.com 
Citation: Musa SAM, Uddin M, Nahian SA, et al. Comparison of initial outcomes between minimally invasive right minithoracotomy and standard median sternotomy in patients undergoing isolated surgical closures of atrial septal defects. Curr Trend Cardiol 2020; 4(1):18-23. 